\title{
SUR UN NOUVEAU SUBULURIDE DE L'ÉCUREUIL XERUS RUTILUS (Cretzchm. 1826)
}

\section{Par Yvonne CAMPANA-ROUGET}

Nous avons eu l'occasion d'examiner un certain nombre de Nématodes parasites d'Ecureuils, de provenances diverses :

$1^{\circ}$ Un mâle récolté chez un Xerus erythropus (Geoffroy 1803) du Niger (Niafunké, 2 février 1928), par Th. Monod, qui nous a été aimablement confié par R.-Ph. Dollfus.

$2^{\circ}$ Une vingtaine d'exemplaires, mâles et femelles, provenant d'un Xerus erythropus du Sénégal mort au Muséum d'Histoire Naturelle de Paris, récoltés par F. Petter en 1952 (1).

$3^{\circ}$ Deux mâles et quatre femelles recueillis chez un Xerus rutilus de Somalie (22 décembre 1948), par E. Biocca, qui nous a laissé le soin de les étudier, ce dont nous le remercions bien vivement.

Tous ces parasites appartiennent au genre Oxynema Linstow 1899 (= Numidica Barreto 1917). Nous n'avons trouvé aucune différence importante entre les deux premiers lots (même hôte, provenance voisine), que nous rattachons avec certitude à $O$. boueti (Gendre 1911).

Par contre, le troisième lot se distingue des précédents par un certain nombre de caractères et nous en faisons une espèce nouvelle : Oxynema bioccai $\mathrm{n}$. sp.

Description. - Corps cylindrique, blanc jaunâtre, atténué aux extrémités. Stries cuticulaires fines, peu visibles. Pas d'ailes latérales.

Bouche hexagonale, sans lèvres, à grand diamètre dorso-ventral (fig. 1, C) ; quatre grandes papilles submédianes, plus ou moins complètement fusionnées. Amphides bien visibles. Cavité buccale profonde, chitinisée, nettement séparée transversalement en deux

(1) Voir dans la Note additionnelle (p. 300) le compte rendu d'autopsie rédigé par F. Petter : en effet, outre les parasites dont il est question ici, l'écureuil hébergeait un Ascaride dont la présence dans l'intestin provoqua une curieuse invagination multiple.

Ann. de Parasitologie, t. XXXI, N ${ }^{\circ} 3 .-1956$. 
parties subégales (35 et $40 \mu$ de haut). Le fond est occupé par les trois dents œsophagiennes que l'on rencontre toujours chez les Subulurides.

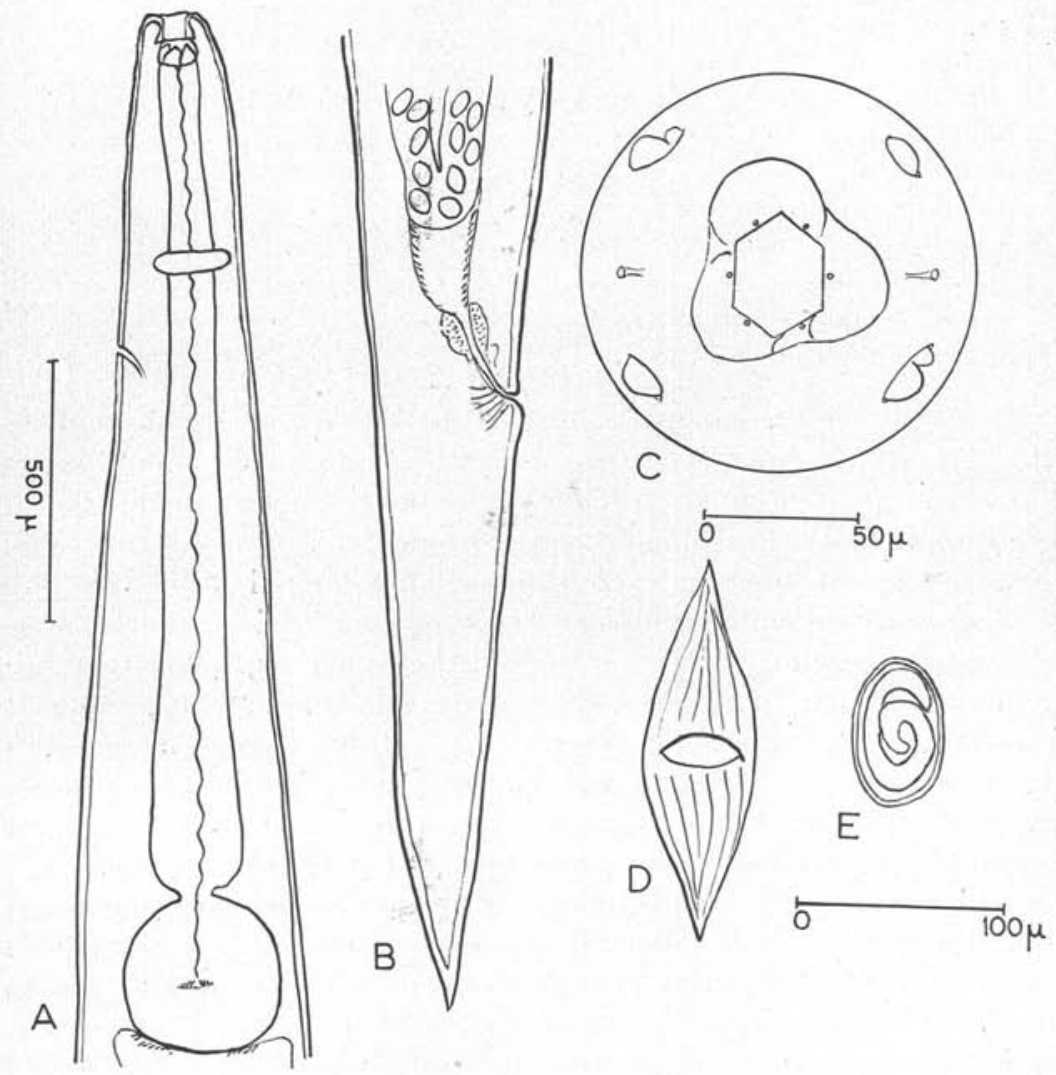

Fig. 1. - Oxynema bioccai, n. sp.

A, Extrémité antérieure, vue latérale. - B, Queue de la femelle, vue latérale. C, Tête, vue apicale. - D, Région vulvaire. - E, Euf embryonné.

Esophage renflé en massue à son extrémité postérieure, séparé par un étranglement profond du bulbe subsphérique (fig. 1, A). Intestin plus large que le bulbe au début, diminuant ensuite légèrement. Queue effilée.

Mâle : 2 exemplaires mesurant 15 et $15,5 \mathrm{~mm}$. Nous décrivons ce dernier :

Ann. de Parásitologie, T. XXXI, N ${ }^{\circ} 3 .-1956$. 
Longueur : 15,5 $\mathrm{mm}$.

Largeur : $0,44 \mathrm{~mm}$. (en avant de la ventouse).

Longueur de l'œsophage : $1,83 \mathrm{~mm}$. (non compris le bulbe).

Longueur du bulbe : $250 \mu$.

Anneau nerveux à $425 \mu$ de l'apex.

Pore excréteur à $520 \mu$ de l'apex.

Diérides non visibles.

Distance du centre de la ventouse au cloaque : $550 \mu$.

Longueur de la ventouse : $165 \mu$.

Spicule droit : $920 \mu$.

Spicule gauche : $640 \mu$.

Gubernaculum : $260 \mu$.

Papilles préanales : 5 paires.

Papilles postanales : 5 paires.

Longueur de la queue : $300 \mu$.

La queue est fortement recourbée sur elle-même, rendant difficile l'étude de la face ventrale. Il n'y a pas à proprement parler d'ailes caudales, mais la cuticule est soulevée au niveau des papilles volumineuses. Le spicule droit, fortement chitinisé, strié transversalement, est ailé sur les $2 / 3$ de sa longueur ; il forme, à $120 \mu$ de l'extrémité distale, une petite expansion triangulaire, puis s'élargit un peu en forme de spatule. Le spicule gauche, très faiblement chitinisé, n’est visible qu'après éclaircissement prolongé ; il est ailé et se termine en pointe. La première paire de papilles préanales, située à la même hauteur que le milieu de la ventouse, lui est franchement externe. La partie externe de la ventouse est formée de stries transversales banales ; la partie centrale est ornée, sur une surface assez bien délimitée, de petits éléments rectangulaires en relief, formant des chaînettes longitudinales très régulières (ce caractère est à peu près constant chez les Oxynema), allant en s'amincissant du centre vers l'extérieur. La lèvre postcloacale, proéminente, présente de petites surélévations du même type, mais moins régulièrement disposées (fig. 2, C et D).

Femelle : 5 exemplaires de 18 à $23,2 \mathrm{~mm}$. Nous décrivons la plus grande :

Longueur : 23,2 $\mathrm{mm}$.

Largeur max. : 0,55 mm.

Longueur de l'œsophage : $1,95 \mathrm{~mm}$.

Longueur du bulbe : $300 \mu$.

Anneau nerveux à $450 \mu$ de l'apex.

Pore excréteur à $630 \mu$ de l'apex.

Queue : 1,35 mm., effilée (Fig. 1, B).

Vulve : $9,7 \mathrm{~mm}$. de l'extrémité antérieure.

Eufs : 75-80 $\mu \times 54-62 \mu$, embryonnés à la ponte (Fig. 1, E). 
La région vulvaire est saillante, allongée en navette et mesure $900 \mu$ de long sur $250 \mu$ de large. L'orifice vulvaire, transversal, a un diamètre de $130 \mu$ (fig. 1, D).

Hôte: Xerus rutilus (Cretzchm.).

Localisation : intestin.

Répartition géographique : Somalie.

Types déposés à l'Institut de Parasitologie de Paris.

Cotypes déposés à l'Institut de Parasitologie de Rome.

Discussion. - Les espèces d'Oxynema sont toutes très voisines, comme le montre le tableau ci-dessous ; elles ne diffèrent guère que par leur taille ou la dimension des spicules, et ces mêmes caractères varient assez largement au sein d'une même espèce. Le nombre de papilles génitales chez le mâle est de 10 ou 11 paires suivant les auteurs ; en réalité, il y en a probablement 10 , la onzième correspondant aux phasmides, plus ou moins visibles suivant les spécimens. La notion d'hôte facilite le diagnostic : il existe une espèce chez la Pintade, O. monodi (Baylis 1930) ; trois espèces chez le Renard, O. crassispiculum (Sonsino 1889), O. numidicata (Seurat 1915) et $O$. alata (Mazhar 1933) (cette dernière est insuffisamment décrite ; d'après la figure, les spicules mesureraient 480 et $270 \mu$ de long et seraient donc plus courts que chez $O$. numidica); une espèce chez un Viverridé, O. suricattæ (Mönnig 1931); une chez un Rongeur, O. typicum Kreis, et deux espèces chez l'Ecureuil, $O$. boueti (Gendre 1911) et $O$. bioccai n. sp. O. boueti a été trouvé, en outre, chez Sciurus annulatus, Lophuromys sikapusi et Praomys tullbergi. Seule, la comparaison directe des deux espèces nous a permis d'établir des caractères différentiels sans risque d'erreur d'interprétation.

Diagnostic différentiel entre $O$. boueti (Gendre 1911) et $O$. bioccai n. sp. - Nos spécimens sont plus grands, les spicules sont plus longs, il n'y a pas d'ailes caudales. Les dernières paires de papilles sont moins rapprochées de l'extrémité caudale et moins proches les unes des autres que chez $O$. boueti (fig. 2, B et $\mathrm{D}$ ). La région périvulvaire affecte une forme de navette. Les deux caractères les plus importants à notre avis sont :

$1^{\circ}$ La forme du spicule droit: il est ailé chez les deux espèces sur les $2 / 3$ de sa longueur, mais, tandis que, chez $O$. boueti (fig. 2, A), il s'amincit régulièrement pour former une pointe mousse, il présente chez $O$. bioccai une expansion triangulaire tot un élargissement terminal en forme de spatule (fig. 2, C). 


\begin{tabular}{|c|c|c|c|c|c|c|c|c|c|c|c|c|c|c|c|}
\hline 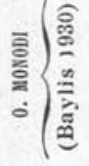 & 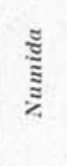 & 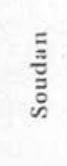 & 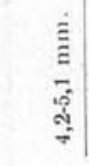 & $\begin{array}{l}0 \\
0 \\
\infty \\
0 \\
0\end{array}$ & & 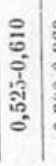 & 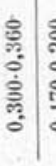 & 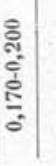 & 10 & $=$ & 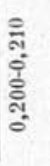 & $\begin{array}{c}\dot{E} \\
\vdots \\
0 \\
\vdots \\
\vdots \\
\vdots \\
-1\end{array}$ & 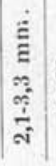 & 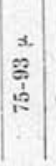 & 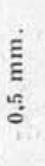 \\
\hline 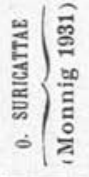 & : & $\begin{array}{l}\bar{\Xi} \\
\dot{D} \\
\Xi \\
\dot{\Xi}\end{array}$ & 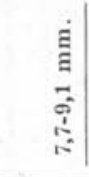 & 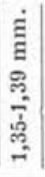 & 1 & 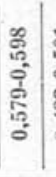 & 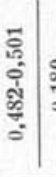 & $\stackrel{\stackrel{\otimes}{\circ}}{=}$ & in & 10 & 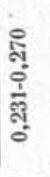 & 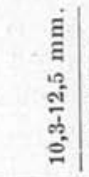 & $\mid \begin{array}{c}\dot{E} \\
\text { E } \\
\hat{\sigma} \\
\hat{b} \\
\dot{0} \\
-\end{array}$ & $\mid \begin{array}{l}5 \\
8 \\
18 \\
x \\
8 \\
8\end{array}$ & 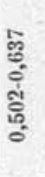 \\
\hline 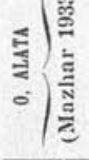 & 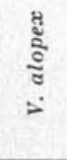 & 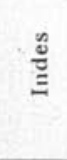 & 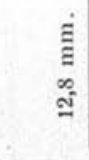 & 1 & 1 & $\frac{\not 0}{0}$ & $\stackrel{\text { : }}{=}$ & $\frac{8}{2}$ & - & 0 & 1 & $\begin{array}{l}\dot{\varepsilon} \\
\Xi \\
\sigma \\
\stackrel{0}{\varrho}\end{array}$ & 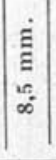 & 1 & 1 \\
\hline 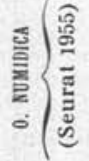 & 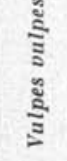 & 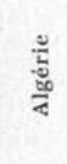 & 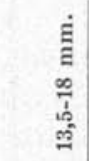 & $\stackrel{\infty}{=}$ & ป్ & : & $\frac{9}{0}$ & $\frac{\mathscr{2}}{6}$ & + & 0 & $\begin{array}{l}\text { సి } \\
\text { సิ }\end{array}$ & 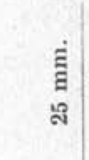 & $\stackrel{\cong}{=}$ & $\begin{array}{l}x \\
\infty \\
0 \\
0 \\
x \\
0 \\
g\end{array}$ & $\begin{array}{l}\text { हี } \\
\text { ลे } \\
\text { ลi }\end{array}$ \\
\hline 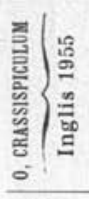 & 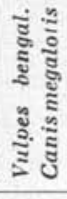 & 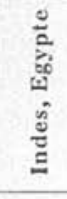 & $\begin{array}{l}\dot{\Xi} \\
\text { है } \\
\stackrel{9}{b}\end{array}$ & สู & 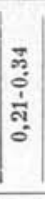 & $\begin{array}{l}5 \\
0 \\
0 \\
0 \\
0 \\
0 \\
0\end{array}$ & $\begin{array}{l}0 \\
0 \\
0 \\
5 \\
0 \\
0\end{array}$ & 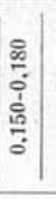 & $\therefore$ & $\begin{array}{l}0 \\
\text { ig } \\
\text { is }\end{array}$ & $\begin{array}{l}\text { कू } \\
\text { ఏ. } \\
\text { के } \\
0\end{array}$ & 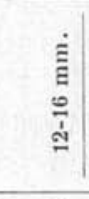 & $\left|\begin{array}{c}\text { a } \\
\text { E } \\
0 \\
5 \\
1 \\
0 \\
15\end{array}\right|$ & $\left|\begin{array}{l}x \\
0 \\
0 \\
0 \\
0 \\
x \\
\infty \\
0 \\
0 \\
0\end{array}\right|$ & \&్ \\
\hline 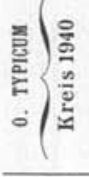 & $\stackrel{5}{\Xi}$ & 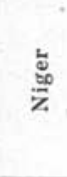 & $\begin{array}{l}\dot{a} \\
\vdots \\
o \\
o \\
\infty \\
0 \\
0 \\
0 \\
0\end{array}$ & 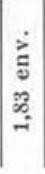 & 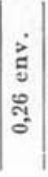 & 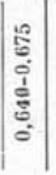 & . & $\begin{array}{l}\frac{5}{0} \\
0 \\
0 \\
0 \\
\frac{0}{0} \\
0\end{array}$ & 10 & in & 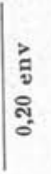 & 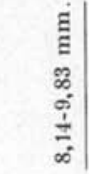 & $\mid \begin{array}{c}\dot{E} \\
\text { ह } \\
\tilde{q}\end{array}$ & $\left|\begin{array}{l}3 \\
\frac{1}{8} \\
1 \\
\frac{0}{4} \\
x \\
5 \\
\frac{1}{2}\end{array}\right|$ & $\stackrel{\sharp}{\circ}$ \\
\hline 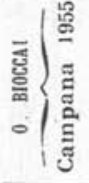 & 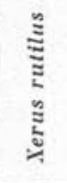 & 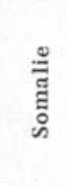 & $\begin{array}{l}\text { E } \\
\text { E } \\
\text { 10 } \\
10 \\
\frac{1}{1} \\
\frac{1}{n}\end{array}$ & $\stackrel{\mathscr{0}}{\stackrel{0}{-}}$ & $\begin{array}{l}\text { 离 } \\
\text { ô. }\end{array}$ & శ్ & 啇 & 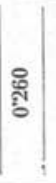 & 10 & in & ஓ్ల్ & 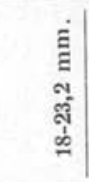 & 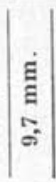 & $\left|\begin{array}{c}3 . \\
0 \\
0 \\
1 \\
15 \\
x \\
0 \\
0 \\
0 \\
10\end{array}\right|$ & $\begin{array}{l}\text { हี } \\
\text { है } \\
\text { है } \\
-\end{array}$ \\
\hline 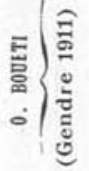 & 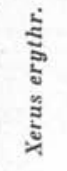 & 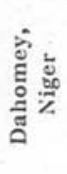 & 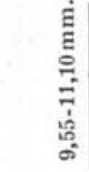 & 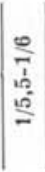 & & 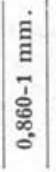 & 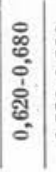 & 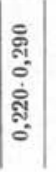 & 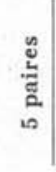 & 总 & 喿 & 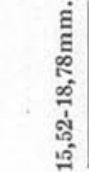 & ลี & $\begin{array}{l}x \\
\text { 으 } \\
x \\
\text { 엉 }\end{array}$ & 总 \\
\hline $\begin{array}{l}\frac{j}{3} \\
\frac{0}{5} \\
\frac{0}{4}\end{array}$ & $\begin{array}{l}\vdots \\
\vdots \\
\stackrel{0}{0}\end{array}$ & $\begin{array}{l}\dot{z} \\
\stackrel{\Xi}{3}\end{array}$ & 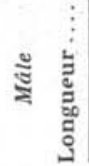 & 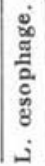 & 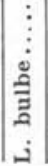 & 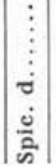 & $\begin{array}{c}\vdots \\
\vdots \\
\vdots \\
\vdots \\
\vdots \\
0\end{array}$ & 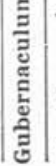 & 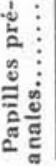 & 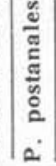 & 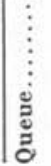 & 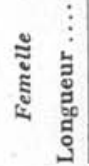 & $\left|\begin{array}{c}n \\
\dot{j} \\
\vdots \\
\vdots \\
\vdots \\
\vdots \\
\dot{n} \\
\frac{0}{0}\end{array}\right|$ & 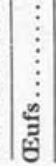 & 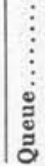 \\
\hline
\end{tabular}


$2^{\circ}$ La position de la première paire de papilles par rapport à la ventouse : située à la même hauteur dans les deux espèces (au niveau du milieu de la ventouse), elle se trouve à la partie externe des

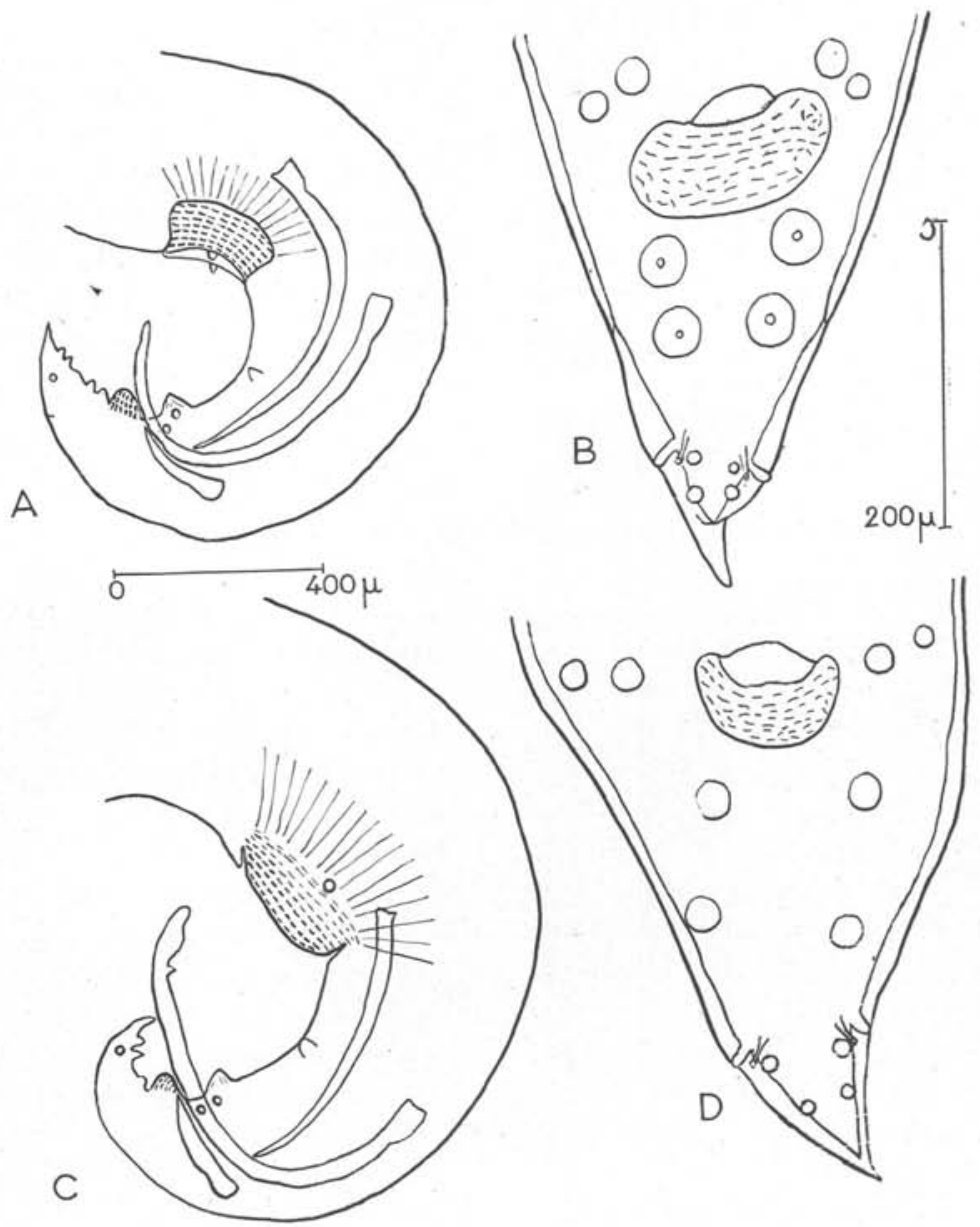

Fig. 2. - A et B, Oxynema boueti, extrémité postérieure du mâle.

C et D, Oxynema bioccai, extrémité postérieure du mâle.

stries longitudinales dans notre matériel, alors qu'elle fait saillie sur le bord interne de l'aire ornementée chez O. boueti. En outre, cette aire est plus nettement délimitée chez $O$. boueti que chez $O$. bioccai où les stries les plus externes deviennent très fines. 
Les affinités de notre espèce avec $O$. crassispiculum sont également très étroites ; cependant, nos spécimens sont plus grands, pour un hôte plus petit, la queue de la femelle est plus courte, celle du mâle a une pointe plus réduite et moins effilée, enfin la forme du spicule droit est différente.

En ce qui concerne O. typicum, les renseignements fournis par Kreis sont trop insuffisants pour que l'on puisse les comparer avec les nôtres : on ne connaît pas l'hôte, on sait seulement que c'est un Rongeur du Niger (et non d'Afrique du Nord, comme le dit l'auteur); il n'est décrit qu'un spicule, les détails de structure de la ventouse ne sont pas donnés. Kreis donne un tableau différentiel avec $\boldsymbol{O}$. crassispiculum et $O$. boueti. Il n'est pas impossible que notre espèce soit identique à la sienne, cependant nos spécimens sont nettement plus grands et la position de la première paire de papilles préanales semble différente.

\section{Révision de la sous-famille des Subulurinæ}

Les différents genres de la sous-famille des Subulurinæ Travassos 1914 sont très proches les uns des autres et la question de leur validité a souvent été mise en discussion. Nous écartons le genre Maupasina Seurat 1913 qui diffère nettement des autres genres et a été placé par Lopez-Neyra (1945) dans la sous-famille des Maupasininæ, puis par Skrjabin, Shikobalova et Mosgovoy (1951) dans la famille des Dubioxyuridx Ortlepp 1937.

Le genre Subulura Molin 1860 est représenté par de très nombreuses espèces parasites d'animaux variés (Oiseaux, Rongeurs, Singes, etc...). Il a été subdivisé par Lopez-Neyra (1944) en Subulura à spicules égaux et Travassallodapa à spicules inégaux; il paraît excessif de créer un genre pour ce seul caractère différentiel.

Le genre Allodapa Diesing 1861 avait été utilisé par Seurat en 1914 pour y classer les espèces suivantes :

Heterakis allodapa Creplin 1853.

Heterakis suctoria Molin 1860 (pro parte).

Allodapa typica Diesing 1861.

Heterakis leprincei Gendre 1909.

Allodapa elongata Seurat 1914.

Allodapa noctux Seurat 1914.

Seurat ajoute en 1915 une espèce parasite de Renard, A. numidica. Les principales différences entre Subulura et Allodapa rési- 
daient, d'après cet auteur, dans la chitinisation plus ou moins prononcée de la capsule buccale et sa division plus ou moins nette en deux parties, ainsi que dans la structure de l'ovéjecteur. Barreto, en 1917 et 1919, estime que ces différences ne sont pas génériques et fait tomber le genre Allodapa en synonymie. Il crée, par contre, le genre Numidica B. 1917 pour la dernière espèce de Seurat, A. numidica, qui possède deux lèvres peu distinctes et pas d'ailes latérales. Il l'oppose au genre Heteroxynema Hall 1916, qui présente deux grandes ailes latérales et trois lèvres nettes. (Ce dernier genre n'a en réalité rien à voir avec les autres et a été placé dans une autre famille).

Le genre Oxynema Linstow 1899 paraissait différer du genre Numidica par la présence d'un seul spicule ; il était représenté par O. crassispiculum (Sonsino 1889) (= O. rectum Linst. 1899), parasite de Renard. On y avait intégré Heterakis boueti Gendre 1911, mais l'auteur, ayant reconnu lui-même que son espèce possédait en réalité deux spicules, elle fut transférée dans le genre Numidica.

Baylis, en 1930, décrit $N$. monodi, parasite de Pintade, et croit, sans pouvoir l'affirmer, à la synonymie des trois genres Subulura, Oxynema et Numidica. En effet, surtout en ce qui concerne les deux derniers, la différence portant sur la présence d'un seul spicule visible chez Oxynema et de deux spicules, dont l'un très faiblement chitinisé, chez Numidica, pouvait être due à une erreur d'interprétation. Cependant, Chertkova, en 1946, redécrit O. crassispiculum chez des Renards du Parc zoologique de Moscou et ne signale qu'un spicule. De son côté, Kreis, en 1940, signale une espèce nouvelle, O. typicum, chez un Rongeur, avec un seul spicule. Il devenait donc impossible de se prononcer sur la validité du genre Numidica, qui s'était enrichi entre-temps d'un certain nombre d'espèces, quand, fort heureusement, Inglis, en 1955, a eu la possibilité d'étudier les types de Linstow et a effectivement trouvé deux spicules. Cet auteur a, en conséquence, établi la synonymie du genre Numidica et reporté chez Oxynema toutes les espèces connues.

Les caractères ayant servi autrefois à différencier Oxynema de Subulura ne sont pas tous valables : c'est ainsi que la division de la capsule buccale en deux parties distinctes et sa forte chitinisation, signalées chez tous les Oxynema, se retrouvent chez nombre d'espèces de Subulura; les lèvres sont généralement au nombre de trois chez les Subulura, mais elles peuvent être subdivisées en 6 ou même 12 lobes; chez les Oxynema, on a signalé deux lèvres peu distinctes portant les papilles pour $O$. numidica (Seurat 1915) et O. suricattæ (Mönnig 1931), mais pas chez les autres espèces. Nous 
avons trouvé une bouche hexagonale, sans lèvres, pour $O$. boueti et $O$. bioccai et nous pensons, en comparant les dessins, qu'il en est de même pour les autres espèces, sans pouvoir l'affirmer, faute de vue apicale.

Un certain nombre de caractères constants permettent cependant d'assurer la validité du genre Oxynema. Ce sont :

$1^{\circ}$ la faible chitinisation du spicule gauche ;

$2^{\circ}$ l'aspect particulier de la ventouse avec sa bordure d'éléments cuticulaires en chainette ;

$3^{\circ}$ la présence sur la lèvre postcloacale, proéminente, de granulations rappelant celles de la ventouse, mais moins régulièrement disposées.

Si le genre est bien défini, il paraît inutile de le ranger dans une sous-famille des Numidinæ Lopez-Neyra 1944.

Le genre Aulonocephalus Chandler 1935 se distingue des précédents par la présence de six lobes péribuccaux, accompagnés ou non d'interlabia. Il comprend deux espèces, A. lindquisti Chandler 1935, parasite d'un Oiseau américain, Callipepla squamata, et A. peramelis (Baylis 1930), parasite d'un Marsupial australien, Perameles obesula.

Skrjabin et Shikobalova, en 1948 , ont créé pour cette dernière espèce le genre Labiobulura, qui ne diffère du précédent que par le nombre de lobes labiaux ; en réalité, les deux espèces possèdent six lobes, mais, chez $A$. peramelis, il y a en outre des interlabia. Nous croyons, comme Chitwood (1950), que l'espèce de Baylis peut rester dans le genre Aulonocephalus, ce qui supprime le genre Labiobulura.

Le genre Leipoanema Johnston et Mawson 1942, représenté par L. ellisi, chez Leipoa ocellata, Australie, est extrêmement différent de tous les autres; la structure buccale est celle d'un Heterakis, avec trois lèvres très développées portant les papilles et une capsule buccale réduite ; par contre, la ventouse ne paraît pas posséder d'anneau chitinoïde. Il serait nécessaire d'étudier plus à fond ce Nématode pour pouvoir le rattacher avec certitude à une famille donnée, mais, à notre avis, il doit être écarté de la sous-famille des Subulurinæ.

Le genre Parasubulura Van den Berghe et Vuylstecke 1938 (= Baylisnumidica Lopez-Neyra 1945) est caractérisé par une ventouse arrondie, renforcée par un anneau musculeux ou chitinoïde. La capsule buccale est divisée ou non en deux parties; les dents œsophagiennes peuvent être très réduites. Il comprend : 
P. gerardi B. et V. 1938, chez Petrodromus tetradactylus. Congo belge.

P. petrodromi (Baylis 1934), chez Petrodromus nigriseta. Tanganyika.

P. callosa (Sandground 1933), chez Petrodromus tetradactylus. Rhodésie du Sud.

Ces trois espèces africaines, parasites de Musaraignes, sont en réalité très voisines. Van den Berghe et Vuylstecke ne sont pas sûrs que l'espèce de Baylis doive entrer dans le genre Parasubula; c'est au contraire $P$. callosa que Skrjabin et coll. (1948), puis Inglis (1955) séparent pour la mettre dans le genre Heterobulura S. et S. 1948. Il nous paraît préférable de conserver seulement le genre Parasubulura pour ces trois espèces.

Van den Berghe et Vuylstecke avaient créé pour ce genre la famille des Parasubuluridæ, intermédiaire entre les Heterakidæ et les Subuluridæ; Inglis (1955) préfère la sous-famille des Parasubulurinæ qui entre alors dans la famille des Subuluridæ.

Nous ne croyons pas nécessaire de conserver cette sous-famille : en effet, le genre Parasubulura ne diffère pas plus du genre Subulura que les autres ; il faudrait alors créer autant de sous-familles que de genres, ce qui complique la systématique sans aucune utilité.

En résumé, nous admettons que la sous-famille des Subulurinæ comprend actuellement les genres suivants :

Subulura Molin 1860.

Oxynema Linstow 1899.

Aulonocephalus Chandler 1935.

Parasubulura Van den Berghe et V. 1938.

\section{Glé de détermination des genres}

1-(6) Ventouse précloacale non pourvue d'un anneau chitinoïde.

2-(5) Bouche à trois lèvres ou sans lèvres.

3-(4) Spicules inégaux ou égaux, bien chitinisés... Subulura.

4-(3) Bouche hexagonale, sans lèvres. Spicules inégaux, dont l'un faiblement chitinisé....... Oxynema.

5-(2) Bouche entourée de 6 lobes, avec ou sans in-

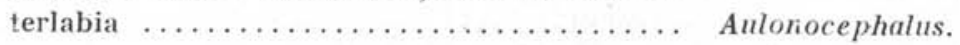

6-(1) Ventouse précloacale pourvue d'un anneau chitinoïde .................... Parasubulura. 


\section{RÉSUMÉ}

Il est décrit un nouveau Subuluride chez un Xerus rutilus de Somalie. Oxynema bioccai n. sp. diffère d'O. boueti (Gendre 1911) par plusieurs caractères, dont les principaux sont la forme du spicule droit et la situation de la première paire de papilles génitales ; elle diffère d'O. crassispiculum (Sonsino 1889) par la forme du spicule droit et la longueur de la queue.

La validité du genre Oxynema par rapport à Subulura est confirmée.

Il est donné une révision de la sous-famille des Subulurinæ: les genres Maupasina Seurat 1913 et Leipoanema Johnston et Mawson 1942 en sont écartés ; les genres Heterobulura et Labiobulura Skrjabin et Shikobalova 1948 sont supprimés. Le genre Parasubulura Van den Berghe et Vuylstecke 1938 est intégré dans les Subulurinæ; les sous-familles des Numidinæ Lopez-Neyra 1944 et des Parasubulurinæ Inglis 1955 sont supprimées.

\section{BIBLIOGRAPHIE}

BARhEto (A. L. de). - 1919. Sobreas especies brasileiras da sub-familia Subulurinæ Travassos 1914. Mem. Inst. Osw. Cruz, XI, p. 10-79.

Baylis (H. A.). - 1930. Mission saharienne Augeras-Draper, 1927-1928. Parasitic Nematodes. Bull. Mus., II, $\mathrm{n}^{\circ} 1$, p. 117-130.

- 1930. Some Heterakide and Oxyuride (Nematoda) from Queensland. Ann. and Mag. Nat. Hist., sẻr. 10, V, p. 354-366.

- 1934. On a Collection of Cestodes and Nematodes from small Mammals in Tanganyika territory. Ann, and Mag. Nat, Hist., XIII, p. 338-353.

Berghe (L. Van den) et Vuylstecke (Cl.). - 1938. Les Parasubuluridæ, famille nouvelle d'Oxyuroidea au Congo belge. Rev. Zool. Bot. Afr., XXXI, p. 376-382.

Chandere (A. C.). - 1935. A new genus and species of Subulurina (Nématodes). Trans. Microsc. Soc., LIV, $\mathrm{n}^{\circ} 1$, p. 33-35.

Chertkova (A. N.). - 1946. Occurence of Oxynema crassispiculum (Sonsino, 1889) in Foxes (en russe). Helminth. storig. Public. Acad. Skrjabin, p. $280-281$.

Gendre (E.). - 1911. Sur quelques espèces d'Heterakis du Dahomey. Extrait des Proc. Verb. Soc. Linnéenne Bordeaux, p. 1-11.

IngL.ıs (W. G.). - 1955. On the family Parasubuluridæe Van den Berghe et Vuylstecke 1938, and the subfamily Numidicine Lopez-Neyra 1945 (Nematoda). Parasitol., XLV, $\mathrm{n}^{\circ}$ 3-4, p. 431-440.

Johnston (H. T.) et Mawson (P. M.). - 1942. Some avian Nematodes from Tailem Bend, South Australia. Trans. Roy. Soc. S. Austr., LXVI, p. 71-73. 
Joyeux (Ch.), Gendre (E.) et Baer (J. G.). - 1928. Recherches sur les Helminthes de l'Afrique Occidentale Française. Coll. Soc. Path. exot., Monographie $I I, 120 \mathrm{p}$.

Kreis (H. A.). - 1940. Beitrage zur Kenntnis parasitischer Nematoden. IX. Parasitische Nematoden aus dem Naturhistorischen Museum Basel. Zentralbl. Bakt. Orig., Band 145, H. 3, p. 163-208.

Linstow (O. von). - 1899. Nematoden aus der Berliner Zoologischer Sammlung. Mitteil. Zool. Samml. Mus. f. Naturk., Berlin, I B., 2 H., p. 1-28.

Lopez-Neyra (C. R.). - 1947. Helmintos de los Vertebrados Ibéricos, Grenade.

Maнzar (A. К.). - 1933-34. On some parasitic Nematodes of Aligarh District. Zeitsch. f. Parasitenk, VI, p. 269-272.

Mönnig (H. O.). - 1931. Two new Nematodes from the Suricat (Viverride). 17th Rep. of Dir. Veter. Serv. and Animal Ind. S. Africa, p. 277-282.

SANDGRound (J. H.). - 1933. Parasitic Nematodes from East Africa and Southern Rhodesia. Bull. Mus. Comp. Zool., LXXV, n 6, p. 263-293.

Seurat (L. G.). - 1913. Sur quelques Nématodes du Sud Tunisien. Bull. Soc. Hist. Nat. Afr. du N., VI, p. 126-130.

1914. Sur quelques Heterakis d'oiseaux. Bull. Soc. Hist. Nat. Afr. du N., VII, p. 195-202.

1915. Sur deux nouveaux parasites du Renard d'Algérie. C.R. Soc. Biol., LXXVIII, p. 122.

Skrjabin (K. I.), Schikobalova (N. P.) et Mosgovoy (A. A.). - 1951. Traité des Nématodes parasites, Skrjabin, Moscou, II, 631 p.

(Institut de Parasitologie, Faculté de Médecine de Paris.

Directeur : Professeur H. Galliard). 